\title{
How Have Multigene Panels Changed the Clinical Practice of Genetic Counseling and Testing
}

\author{
Robert Pilarski, MS, LGC, MSW ${ }^{1}$
}

\begin{abstract}
Historically, genetic testing (and billing) for hereditary cancer risk was essentially performed gene by gene, with clinicians ordering testing only for the genes most likely to explain a patient's or family's cancer presentation, with laboratories typically charging $\$ 1,000$ to $\$ 1,500$ for each gene that was sequenced. Given the expense, only patients at high risk of having a hereditary syndrome were offered testing. With the introduction of next-generation sequencing technologies, however, laboratories are able to test for multiple genes at the same time with greater efficiency, significantly decreased costs, and relatively little increased expense when adding additional genes. This has drastically altered clinical practice so that clinicians now typically order testing for a panel of multiple genes for most patients. Although this approach has streamlined the diagnostic odyssey, it has introduced several problems, as well, including difficulties in choosing the appropriate panel test for a given patient, assessing the significance of identified genetic variants (including variants of uncertain significance [VUS]), and understanding the disease risks and management associated with pathogenic variants in a given gene. Many laboratories offer testing for genes that have limited data supporting their associated cancer risks, which then leads to an inability to set management guidelines based on that gene. In addition, testing larger numbers of genes increases the likelihood of finding one or more VUS, which introduce their own management issues. Thus, although panel testing has certainly moved clinical practice forward in many ways, it has also raised its own set of problems that increase the complexity of genetic counseling and highlight the need for education of community practitioners on the complexities and nuances of this testing. Whenever possible, testing should be performed by, or in consultation with, cancer genetics professionals.
\end{abstract}

J Natl Compr Canc Netw 2021;19(1):103-108 doi: 10.6004/jnccn.2020.7674

${ }^{1}$ Division of Human Genetics, Department of Internal Medicine, James Comprehensive Cancer Center, The Ohio State University, Columbus, Ohio.
Historically, genetic testing was essentially performed gene by gene. Based on existing technologies, sequencing was set up and optimized in separate assays for each gene for which a laboratory offered testing. As a result, billing was also performed on a gene-by-gene basis, with laboratories typically charging $\$ 1,000$ to $\$ 1,500$ or more per gene. For example, testing for the BRCA1 and BRCA2 genes cost $\$ 3,300$. Qualification for insurance coverage for testing was also based on the specific gene being tested, although until recently such criteria were only set for a few genes, such as BRCA1, BRCA2, APC, and the Lynch syndrome genes. Given this, genetic testing was only offered to select patients at highest risk of having a pathogenic variant (mutation), and only for the genes in which they were most likely to have a variant. Positive results were thus usually consistent with the cancer presentation in a family and offered relatively clear management options because generally testing was only offered for genes with clearly defined risks and management. Some have argued, however, that this complicated process also unfortunately led to lengthening of the diagnostic process. ${ }^{1}$

In 2010, however, the introduction of nextgeneration sequencing (NGS) technologies into clinical laboratory practice drastically altered the testing landscape. ${ }^{2}$ With NGS, multiple genes can be sequenced at the same time, leading to significantly decreased time, labor, and costs for testing. This led to the introduction of multigene panel testing, in which multiple genes are grouped together and offered as a single test, with the types and numbers of genes offered and the types of panels they are grouped into varying by laboratory. At the same time, there has been a broadening of the range of patients who are offered testing, and the types of genes for which they are tested. For example, current NCCN Clinical Practice Guidelines in Oncology (NCCN Guidelines) for Genetic/Familial High-Risk Assessment: Breast, Ovarian, and Pancreatic recommend offering genetic testing to (among others) all patients with exocrine pancreatic, ovarian, high-risk/metastatic prostate and male breast cancers, and all female patients with breast cancer diagnosed at age $\leq 45$ years (as well as patients 
meeting other criteria addressing personal and family history). ${ }^{3}$ Using multigene panels, some studies suggest that up to $17 \%$ of high-risk patients testing negative for $B R C A$ pathogenic variants will have a pathogenic variant identified in another gene. ${ }^{4,5}$ In many centers, patients have the option of undergoing testing for genes related to cancers that are not present and/or are not genetically linked to the cancers in their family. In addition, the increasing availability of cancer treatments directed to patients with specific gene defects (eg, PARP inhibitors for patients with mutations in the BRCA genes; immune checkpoint inhibitors in patients with Lynch syndrome) and growing awareness of broader ranges of tumors caused by specific hereditary syndromes have also increased the interest in genetic testing among patients and providers.

Although this approach has streamlined the diagnostic odyssey and decreased testing costs, it has also introduced many problems, including difficulties in choosing the appropriate panel test for a given patient, assessing the significance of identified genetic variants (including variants of uncertain significance [VUS]), and understanding the disease risks and management associated with pathogenic variants in a given gene. This review discusses each of these in more detail.

\section{Choosing a Panel}

There are a number of ways in which genes are grouped into panels. In disease-specific panels, testing is only offered for genes associated with a given cancer. For some laboratories this includes only genes with a proven association, whereas others may also include genes with suspected but unconfirmed associations with the cancer type in question (sometimes called limited evidence genes). Examples would be panels specific to breast cancer (offering up to $\geq 18$ genes) or colon cancer (offering $\geq 20$ genes). In guidelines-based panels, testing is only offered for genes that have clearly established management guidelines. Typically, these panels are also grouped by disease type, but may exclude genes included in disease-specific panels that do not have established management guidelines. A guidelines-based breast cancer panel, for example, might include only 6 to 8 genes. In pan-cancer or comprehensive panels, testing is offered for a large group of genes (typically 35-85 genes or more) that are known or suspected to be associated with a broad range of cancer types. Each of these panel types has its own advantages and disadvantages.

\section{Disease-Specific Panels}

With disease-specific panels, any pathogenic variant found is to be expected because only genes related to the cancers seen in the family are being tested, and thus an answer has been provided to the patient/family by a positive result. Therefore, there are rarely surprises of finding a pathogenic variant in an unrelated gene (see "Comprehensive Cancer Panels" section). There are, however, several downsides to these types of panels. First, many genes are associated with increased risks for multiple different cancers and it may be difficult to tie them to a single cancer type. Second, some genes may not be clearly linked to a particular cancer now but may be shown to be so by future research, and thus leaving them off a panel could represent a missed opportunity for the patient. Current examples include possible (but not yet definitive) associations between the Lynch syndrome genes and breast and prostate cancers, and between the $R A D 51 C, R A D 51 D$, and other genes and breast cancer. $^{6-8}$ Lastly, genes may be included on diseasespecific panels that do not have clear management guidelines, such as the inclusion of $H O X B 13$ on prostate cancer panels or PALB2 on pancreatic cancer panels. Although numerous studies support $\mathrm{HOXB13}$ as a prostate cancer gene, the degree of risk it confers is still uncertain, and thus management guidelines have not been established. ${ }^{9,10}$ For PALB2, management guidelines for pancreatic cancer have only recently been established despite testing being available for some time (in part because of the significantly increased breast cancer risks associated with it).

\section{Guidelines-Based Panels}

Guidelines-based panels may be the most conservative, in that any gene in which a pathogenic variant is found will have relatively clear management guidelines that can be provided to the family, at least for the main cancers caused by that gene. The downsides to these types of panels include that they may leave off genes that are clearly felt to increase risk for a given cancer but for which there are no established management guidelines because of lack of sufficient data (as noted above with prostate cancer risk with $H O X B 13$ and pancreatic cancer risk with PALB2). Also, some of the genes included on these panels may also increase risk for other cancers that do not have management recommendations.

\section{Comprehensive Cancer Panels}

Comprehensive cancer panels generally include most or all cancer genes for which a laboratory offers testing. Clinicians may prefer these types of panels due to mounting evidence showing that a subset of patients will be found to have a pathogenic variant in a gene that has not been associated with the cancers in their family, and that some of these variants will be clinically actionable. ${ }^{11}$ Comprehensive panels also reduce the need to retest a patient if unsuspected cancer associations are later identified for a specific gene that was not included on a disease-specific panel. Furthermore, some patients wish 
to be tested for any known or suspected cancer risk, even if unrelated to the cancers in their family, as long as it does not significantly affect the price they will pay for testing. On the downside, large panels significantly increase the likelihood of finding a VUS (see next section) and of finding a pathogenic variant in a gene that is not consistent with the family history, yet presents significant management implications (see "Management Issues" section). ${ }^{12,13}$

\section{Variants of Uncertain Significance}

A VUS is essentially a change in a gene that cannot be classified as either disease-causing or benign based on the available evidence. In current practice, patients and their providers are informed of these, but are strongly advised not to base medical management on the presence of the VUS. For some patients and providers, ignoring a change in a gene known to increase cancer risk is easier said than done, and anxiety and confusion arise over the possible unknown risks the patient faces and the inability to screen for them. ${ }^{14}$ In addition to increasing distress, this could also potentially lead to misuse of healthcare dollars by patients seeking cancer screening for an unproven risk. Although there is a possibility of finding a VUS with even a single-gene test, the likelihood of finding $\geq 1$ of these increases with the number of genes that are analyzed..$^{15}$ Large panels may sometimes identify $\geq 2$ VUS in a single person. Of note, VUS are more common in non-White patients. Patients need to be made aware of these points, because those who would be distressed by the finding of a VUS may want to choose the smallest panel that meets their medical indication based on their personal and family history.

To complicate matters further, laboratories may differ in their interpretation of the same genetic variant based on their assessment of the available data, and in some cases, based on differing weight that they put on such data in their proprietary algorithms for classifying variants. ${ }^{16}$ In some cases this can lead to various family members receiving different test interpretations of the same variant, depending on the laboratory that performed their testing.

\section{Management Issues}

As noted earlier, for many genes offered on broader panels, data are not robust enough to support management recommendations, and identification of a pathogenic variant does not alter treatment recommendations that would have been made based on the personal/family history. Even for well-understood genes, management of cancer risk based on genetic test results is becoming more complex, along with the increasing understanding that not all pathogenic variants in a gene necessarily carry the same risk. Although for most genes the specific management recommendations do not vary based on the specific variant, there are notable exceptions. For the RET gene, which causes medullary thyroid cancer, various pathogenic variants are grouped into different risk groups and management varies accordingly. ${ }^{17}$ For the CHEK2 gene, which causes breast and other cancers, most data are from patients with the 1100delC founder variant, and management recommendations for other pathogenic variants in CHEK2 are extrapolated from this. ${ }^{3}$ However, missense variants such as the I157T variant appear to lead to lower breast cancer risks (and uncertain colon cancer risks), although specific management recommendations have not been made for it. ${ }^{3,18-20}$ For the $N B N$ gene, current data suggest that only the 657del5 Slavic founder variant may be associated with increased breast cancer risk, although evidence is mixed. ${ }^{3}$

In addition, an increasing number of genes offered on panel tests are moderate-penetrance genes that pose a lower risk for cancer than high-risk genes such as $B R C A 1$ and BRCA2. It is suspected that the cancer risks from these genes are often modified by other genetic or environmental factors, so that the pathologic variant alone does not entirely determine the patient's risk. This has been demonstrated for CHEK2 and PALB2, in which pathologic variant carriers with stronger family histories of breast cancer have higher risks for breast cancer than those with weaker or no family history. ${ }^{21,22}$ It should be noted that genetic modifiers have even been identified for high-risk genes such as $B R C A 1$ and $B R C A 2$ that may help explain differences in cancer risks found between higher-risk clinical patient cohorts versus populationbased cohorts. ${ }^{23}$ Extensive research is currently underway to investigate the clinical utility of examining the impact of multiple modifying genes on cancer risk-the so-called polygenic risk scores. ${ }^{24}$ The converse of this is that it is at least theoretically possible that a relative who has not inherited the known family mutation in one of these genes may still be at some degree of increased risk due to some of the other risk factors that are shared in the family.

Equally complicated are results that are inconsistent with the cancer presentation in a family yet that carry significant management implications. For example, patients from families with gastric cancer with pathogenic variants in the $\mathrm{CDHl}$ gene are advised to undergo prophylactic gastrectomy given the significant risk for diffuse gastric cancer that cannot be identified though screening. ${ }^{25}$ Because pathogenic variants in $\mathrm{CDH} 1$ also increase the risk for lobular breast cancer, this gene is included on most breast cancer panels. Yet there is understandable anxiety on the part of both provider and patient in recommending gastrectomy when a pathogenic variant is found in a family that has breast cancer 
but no gastric cancer present in multiple generations. Although evidence exists that early diffuse gastric cancer has been identified in such patients who do undergo prophylactic gastrectomy, there is still some uncertainty regarding how best to manage these families. ${ }^{26,27}$

\section{Additional Issues}

\section{Choosing a Laboratory}

Although there are many well-established genetic testing laboratories, there are also an increasing number of newer laboratories with variable degrees of scientific and methodologic soundness. Additionally, even for wellestablished laboratories, there may be significant differences in testing methodologies in general or for specific genes, as well as in their customer service policies (discussed in the next section). Thus, a number of issues need to be considered in choosing a laboratory, such as types and numbers of gene and panel tests offered; qualification and accreditation of the laboratory directors, staff, and laboratory; years of experience and volume of testing; specific testing methodologies and depth of coverage of their NGS testing; rigor of their variant classification process; and policies on sharing variant data with publicly accessible databases, such as ClinVar, and on recontacting providers when they have reclassified a variant.

\section{Pricing/Insurance Billing/Financial}

Assistance Policies

Another issue to consider is cost of the test to the patient and healthcare system. List prices for panel tests in different laboratories vary from approximately $\$ 1,500$ to $\$ 4,000$ and higher. With contracts, the amount actually billed to insurers can vary dramatically from this and may be difficult to determine. The cost to the patient can also be confusing. If the laboratory is contracted with their insurer and the test is covered by their insurance, patients may be responsible for only the same copay costs they pay for other laboratory tests. But in other cases, they may face significantly higher costs. Some laboratories will conduct an insurance preauthorization or benefits investigation and notify the patient of their estimated out-of-pocket costs, at which point the patient may decline the test if it is too expensive. Many laboratories offer financial assistance programs to help limit patient costs, and/or offer a selfpay price of approximately $\$ 250$ to $\$ 400$ to patients whose insurance does not cover testing or whose costs through insurance would be excessive. Policies differ significantly among laboratories, however, and providers should compare across laboratories and familiarize themselves with the policies of the laboratory they choose to use. In most cases patients should not be encountering out-of-pocket costs of several thousands of dollars as they did in the past.

Insurers have generally been reluctant to pay for panel tests because of the undocumented clinical benefit of testing for many of the genes included on panels. In general, most insurers are technically only paying for the $B R C A$ or Lynch syndrome genes included on panels, and the laboratories absorb the cost of testing for the additional genes. Therefore, for most insurers, eligibility for genetic testing coverage is based on meeting their testing criteria for BRCA or Lynch syndrome. More recently, some insurers have added criteria for a few additional cancer genes as well.

A new twist in the genetic testing landscape is sponsored testing. In these programs, a laboratory partners with a biomedical company to offer free testing to patients with specific diseases in exchange for the biomedical company obtaining access to deidentified patent data and test results to further their own research and development. Recently, for example, such testing has been made available by one laboratory to patients with pancreatic and prostate cancers.

Given the complexity of these issues, nongenetics providers are encouraged to consult with the genetic counselors/providers in their area for discussion and assistance.

\section{Somatic (Tumor) Testing}

Although beyond the focus of this article, it should be noted that testing tumors for somatic mutations that were acquired as the tumor developed is an increasingly common part of oncology practice. Identification of mutations in various genes can direct the choice of chemotherapy in specific patients (so-called precision medicine), and many laboratories offer tumor testing services. In some cases, testing the tumor also provides evidence of the presence of a germline mutation in the patient that needs to be addressed with germline genetic testing and counseling. ${ }^{28}$

\section{Community Education}

Although panel testing has certainly moved clinical practice forward in many ways, it has also introduced its own set of problems that highlight the need for education of community practitioners on the complexities and nuances of this testing. As discussed earlier, providers must consider numerous issues when choosing a laboratory and panel for genetic testing, and patients should undergo pretest counseling addressing the risks, benefits, and limitations of this testing. Ideally, counseling and testing should be performed by genetics professionals with experience in cancer genetics. ${ }^{29}$ However, given the shortage of genetic counselors in the United States and the variability in access by geographic region and institution, this is not always possible. ${ }^{30,31}$ Laboratories have begun offering direct-to- 
consumer and self-directed testing options to patients that circumvent or replace the need for traditional pretest and posttest counseling. At the same time, various alternative service delivery models are being explored to address the provider shortage. In some, online services such as prerecorded educational videos and chat-bots are being used. In others, community oncology providers perform streamlined genetic testing and/or collaborate with genetics centers to offer testing to their patients in straightforward cases, with referral to their genetics colleagues for complex cases. ${ }^{32-36}$ Cases may qualify as complex because they are clinically challenging or because there are psychologic issues or family dynamics that require more time and counseling than the oncology practitioner is prepared to provide. Despite the move to encourage these alternative models, studies indicate that community practitioners are in general not well-equipped for this and are in need of significant education and support to participate in the provision of cancer genetics services in this way. ${ }^{37,38}$

\section{Conclusions}

NGS and the use of multigene panel testing have dramatically altered the practice and costs of clinical genetic testing for hereditary cancer risk. However, this is accompanied by a corresponding increase in both the complexity of the testing and the information that must be conveyed to patients in pretest and posttest counseling, highlighting the need for increased genetics education for oncology providers so that they can play a role in the provision of these services. Regardless of the service delivery model used, all patients undergoing genetic testing should be counseled regarding the risks, benefits, and limitations prior to testing.

Submitted August 17, 2020; accepted for publication October 20, 2020

Disclosures: The author has disclosed that he has no financial interests, arrangements, or affiliations with the manufacturers of any products discussed in this article or their competitors.

Correspondence: Robert Pilarski, MS, LGC, MSW, Ambry Genetics, 1 Enterprise, Aliso Viejo, CA 92656. Email: rpilarski@ambrygen.com

\section{References}

1. Mannelli M, Castellano M, Schiavi F, et al. Clinically guided genetic screening in a large cohort of Italian patients with pheochromocytomas and/or functional or nonfunctional paragangliomas. J Clin Endocrinol Metab 2009;94:1541-1547.

2. Gomy I, Diz MP. Hereditary cancer risk assessment: insights and perspectives for the next-generation sequencing era. Genet Mol Biol 2016; 39:184-188.

3. Daly MB, Pal T, Berry MP, et al. NCCN Clinical Practice Guidelines in Oncology: NCCN Guidelines for Genetic/Familial High-Risk Assessment: Breast, Ovarian, and Pancreatic. Version 2.2021. Accessed November 24, 2020. To view the most recent version, visit NCCN.org

4. Walsh T, Casadei S, Coats $\mathrm{KH}$, et al. Spectrum of mutations in BRCA1, BRCA2, CHEK2, and TP53 in families at high risk of breast cancer. JAMA 2006;295:1379-1388.

5. Walsh T, Lee MK, Casadei S, et al. Detection of inherited mutations for breast and ovarian cancer using genomic capture and massively parallel sequencing. Proc Natl Acad Sci USA 2010;107:12629-12633.

6. Kohlmann W. Lynch syndrome and breast cancer risk: weighing the data. JCO Precis Oncol 2020;4:128-130.

7. Wood ME, McKinnon W, Garber J. Risk for breast cancer and management of unaffected individuals with non-BRCA hereditary breast cancer. Breast J 2020;26:1528-1534.

8. Beebe-Dimmer JL, Kapron AL, Fraser AM, et al. Risk of prostate cancer associated with familial and hereditary cancer syndromes. J Clin Oncol 2020;38:1807-1813.

9. Isaacs WB, Cooney KA, Xu J. Updated insights into genetic contribution to prostate cancer predisposition: focus on HOXB13. Can J Urol 2019; 26(5 Suppl 2):12-13.

10. Xu J, Lange EM, Lu L, et al. HOXB13 is a susceptibility gene for prostate cancer: results from the International Consortium for Prostate Cancer Genetics (ICPCG). Hum Genet 2013;132:5-14.

11. Desmond A, Kurian AW, Gabree M, et al. Clinical actionability of multigene panel testing for hereditary breast and ovarian cancer risk assessment. JAMA Oncol 2015;1:943-951.

12. Chang J, Seng S, Yoo J, et al. Clinical management of patients at risk for hereditary breast cancer with variants of uncertain significance in the era of multigene panel testing. Ann Surg Oncol 2019;26:3389-3396.

13. Tsaousis GN, Papadopoulou E, Apessos A, et al. Analysis of hereditary cancer syndromes by using a panel of genes: novel and multiple pathogenic mutations. BMC Cancer 2019;19:535.
14. Mighton C, Shickh S, Uleryk E, et al. Clinical and psychological outcomes of receiving a variant of uncertain significance from multigene panel testing or genomic sequencing: a systematic review and meta-analysis [published online September 14, 2020]. Genet Med. doi: 10.1038/ s41436-020-00957-2

15. Dominguez-Valentin $\mathrm{M}$, Nakken $\mathrm{S}$, Tubeuf $\mathrm{H}$, et al. Results of multigene panel testing in familial cancer cases without genetic cause demonstrated by single gene testing. Sci Rep 2019;9:18555.

16. Amendola LM, Jarvik GP, Leo MC, et al. Performance of ACMG-AMP variant-interpretation guidelines among nine laboratories in the Clinical Sequencing Exploratory Research Consortium. Am J Hum Genet 2016;99:247.

17. Wells SA Jr, Asa SL, Dralle H, et al. Revised American Thyroid Association guidelines for the management of medullary thyroid carcinoma. Thyroid 2015;25:567-610.

18. Liu C, Wang QS, Wang YJ. The CHEK2 I157T variant and colorectal cancer susceptibility: a systematic review and meta-analysis. Asian Pac J Cancer Prev 2012;13:2051-2055.

19. Liu C, Wang Y, Wang QS, et al. The CHEK2 I157T variant and breast cancer susceptibility: a systematic review and meta-analysis. Asian Pac J Cancer Prev 2012;13:1355-1360.

20. Kilpivaara O, Vahteristo P, Falck J, et al. CHEK2 variant I157T may be associated with increased breast cancer risk. Int J Cancer 2004;111:543-547.

21. Gronwald J, Cybulski C, Piesiak W, et al. Cancer risks in first-degree relatives of $\mathrm{CHEK} 2$ mutation carriers: effects of mutation type and cancer site in proband. Br J Cancer 2009;100:1508-1512.

22. Antoniou AC, Foulkes WD, Tischkowitz M. Breast-cancer risk in families with mutations in PALB2. N Engl J Med 2014;371:1651-1652.

23. Peterlongo $P$, Chang-Claude J, Moysich KB, et al. Candidate genetic modifiers for breast and ovarian cancer risk in BRCA1 and BRCA2 mutation carriers. Cancer Epidemiol Biomarkers Prev 2015;24:308-316.

24. Kuchenbaecker KB, McGuffog L, Barrowdale D, et al. Evaluation of polygenic risk scores for breast and ovarian cancer risk prediction in BRCA1 and BRCA2 mutation carriers. J Natl Cancer Inst 2017;109:djw302.

25. van der Post RS, Vogelaar IP, Carneiro F, et al. Hereditary diffuse gastric cancer: updated clinical guidelines with an emphasis on germline $\mathrm{CDH} 1$ mutation carriers. J Med Genet 2015;52:361-374

26. Huynh JM, Laukaitis CM. Panel testing reveals nonsense and missense $\mathrm{CDH} 1$ mutations in families without hereditary diffuse gastric cancer. Mol Genet Genomic Med 2016;4:232-236. 
27. Hamilton JG, Long JM, Brandt AC, et al. Patients' medical and psychosocial experiences after detection of a $\mathrm{CDH} 1$ variant with multigene panel testing. JCO Precis Oncol 2019;3:1-14

28. Cheng $\mathrm{HH}$, Sokolova AO, Schaeffer EM, et al. Germline and somatic mutations in prostate cancer for the clinician. J Natl Compr Canc Netw 2019;17:515-521.

29. Pal T, Lee JH, Besharat A, et al. Modes of delivery of genetic testing services and the uptake of cancer risk management strategies in BRCA1 and BRCA2 carriers. Clin Genet 2014;85:49-53.

30. Hoskovec JM, Bennett RL, Carey ME, et al. Projecting the supply and demand for certified genetic counselors: a workforce study. J Genet Couns 2018;27:16-20.

31. Knapke S, Haidle JL, Nagy R, et al. The current state of cancer genetic counseling access and availability. Genet Med 2016;18: 410-412.

32. Cohen SA, Nixon DM. A collaborative approach to cancer risk assessment services using genetic counselor extenders in a multisystem community hospital. Breast Cancer Res Treat 2016;159: 527-534.
33. Cohen SA, Bradbury A, Henderson V, et al. Genetic counseling and testing in a community setting: quality, access, and efficiency. Am Soc Clin Oncol Educ Book 2019;39:e34-44.

34. Powell CB, Laurent C, Ciaravino G, et al. Streamlining genetic testing for women with ovarian cancer in a Northern California health care system. Gynecol Oncol 2020;159:221-228.

35. Colombo N, Huang G, Scambia G, et al. Evaluation of a streamlined oncologist-led brca mutation testing and counseling model for patients with ovarian cancer. J Clin Oncol 2018;36:1300-1307.

36. Ormond KE, Hallquist MLG, Buchanan AH, et al. Developing a conceptual, reproducible, rubric-based approach to consent and result disclosure for genetic testing by clinicians with minimal genetics background. Genet Med 2019;21:727-735.

37. Blazer KR, Nehoray B, Solomon I, et al. Next-generation testing for cancer risk: perceptions, experiences, and needs among early adopters in community healthcare settings. Genet Test Mol Biomarkers 2015;19:657-665

38. O'Brien MA, Carroll JC, Manca DP, et al. Multigene expression profile testing in breast cancer: is there a role for family physicians? Curr Oncol 2017;24:95-102. 